\title{
Surface curing effect on reflection response of hardened cementitious mortar samples
}

\author{
Ugur Cem Hasar a,*, Mucahit Izginli a,b, Hamdullah Ozturk ${ }^{\mathrm{a}}$, Huseyin Korkmaz a, \\ Abdulkadir Cevik ${ }^{\mathrm{c}}$, Mohammad Refaat Irshidat ${ }^{\mathrm{d}}$ \\ ${ }^{a}$ Department of Electrical and Electronics Engineering, Gaziantep University, 27310 Gaziantep, Turkey \\ ${ }^{\mathrm{b}}$ Department of Electrical and Electronics Engineering, Hasan Kalyoncu University, Gaziantep 27410, Turkey \\ ' Department of Civil Engineering, Gaziantep University, 27310 Gaziantep, Turkey \\ ${ }^{\mathrm{d}}$ Center for Advanced Materials, Qatar University, Doha, 2713, Qatar
}

\section{A R T I C L E I N F O}

\section{Keywords:}

Cementitious mortar

Sample curing

Propagation-related research

Hardening

\begin{abstract}
A B S T R A C T
Surface curing effect of hardened engineering cementitious mortar (ECM) samples with distinct water-to-binder ratios $(w / b)$ is analyzed by recording amplitude of reflection response $|\Gamma|$ or conductance term $g$ measured by a microwave open-ended waveguide probe. Frequency dependencies (between 3.95-5.85 GHz) of $|\Gamma|$ and $g$ measured from various surfaces (top, bottom, and lateral) of ECM samples with $w / b=0.2$ and 0.3 are presented at the 720th days of curing (hardened ECM). In addition, their temporal dependencies (over 1-9 and 720-815 days) are presented for the frequency value of $4.56 \mathrm{GHz}$. From our analysis, contrary to top and lateral surface behaviors, it is noted that crossover between $g$ values measured from bottom surfaces of ECM samples with $w / b=0.2$ and 0.3 delays for a long time (around 550 days). Propagation-related studies should take into account reflection profiles varying with wall height for accurate prediction.
\end{abstract}

\section{Introduction}

Cement-based materials (CBMs) are the most widely employed construction materials in earth. However, its brittleness limits their application for areas vulnerable to strong earthquakes and thus calls for new construction materials resistant to earthquakes. On the other hand, engineered cementitious composites (ECCs) are a special kind of fiber reinforced CBMs [1] to meet the demand for construction areas prone to strong earthquakes, because these composites have a high ductility compared with that of CBMs. Besides, ECCs are unique composites having high tensile and flexural strengths thanks to the fiber which interacts with cement matrix, resulting in crack size minimization [2].

Microwave non-destructive testing methods have shown great potential in recent years for structural health monitoring of CBMs [3-8] and ECCs [9-13] by establishing a link between their electromagnetic properties and physical, mechanical, and chemical properties. In studies $[4,5]$, water-to-cement and sand-to-cement ratios, which are the two important factors affecting compressive and flexural strengths, within CBMs were determined by using microwave near-field open-ended waveguide measurements. Besides, microwave reflection properties of fresh concrete and mortar specimens were measured by near-field openended waveguide measurements $[8,14]$. On the other hand, the relation between microwave reflection properties and compressive strength and thus prediction of strength, modulus of rupture, and modulus of elasticity of ECCs were examined in recent studies [9-12].

In addition to above studies establishing a link between microwave signals and non-electrical properties of CBMs and ECCs, our main concern in this study is to examine surface shielding effects on their reflection and transmission properties, because such studies not only are useful for structural health monitoring of these materials but also are required in propagation-related research; e.g., wireless local area networks [15]. To this end, the effect of shielding on crossover times between reflection responses of mortar specimens (CBMs) with diverse mixture proportions of water and cement was examined by microwave a far-field free-space method [6]. Similarly, the effect of surface placement on transmission properties of hardened mortar samples (CBMs) was investigated in the study [7]. The above studies were restricted to CBMs. To examine the effect of different curing conditions for ECCs, in a recent study, the influence of specimen curing conditions on reflection response of fresh engineered cementitious mortar (ECM) specimens was examined [13]. In this study, we examine the effect of sample curing conditions on reflection response of hardened ECM sample using microwave near-field open-ended waveguide measurements. It has been demonstrated for the first time in the literature that the crossover

\footnotetext{
* Corresponding author.

E-mail address: uchasar@gantep.edu.tr (U.C. Hasar).
} 
Table 1

Raw material proportions of the ECM samples.

\begin{tabular}{lllllll}
\hline Sample & Cement (\%) & Fly Ash (\%) & Sand & Water & w/b & HRWR \\
\hline M1 & 1.00 & 1.20 & 0.80 & 0.44 & 0.20 & 0.016 \\
M2 & 1.00 & 1.20 & 0.80 & 0.66 & 0.30 & 0.008
\end{tabular}

between the values of $g$ values measured from bottom surfaces of ECM samples with $w / b=0.2$ and 0.3 takes place around 550 days of curing (according to the fitted data), while the crossover between $g$ values measured from top (or lateral) surfaces of ECM samples with $w / b=0.2$ and $w / b=0.3$ occurs very early (before the 10th day of curing). Besides, it is also shown that different reflection profiles should be accounted for accurate prediction in propagation-related studies.

\section{Sample preparation and curing conditions}

Two mixes were used to prepare the ECM samples in this study. Table 1 presents mixture proportions of these mixes [9,13]. Silica sand with $100 \mu \mathrm{m}$ mean size and ordinary Portland cement (ASTM TypeI) were used in preparation of these samples [16]. Samples with two different $w / b$ values ( $w$ denotes water mass, $b=m_{c}+m_{f}$, and $m_{c}$ and $m_{f}$ denote masses of cement and fly ash) were examined to analyze the influence of water amount on reflection response of hardened ECM samples. High range water reducer (HRWR) was mixed with each sample to improve their workability. Three sets of each mortar sample were prepared in cubical forms $(15 \mathrm{~cm} \times 15 \mathrm{~cm} \times 15 \mathrm{~cm})$ to reduce measurement errors. After the removal from their molds (after one day), their surfaces were furbished with a sandpaper to improve the accuracy of open-ended waveguide measurements. Bottom of each sample was set to the ground before, during, and after all measurements in our microwave laboratory (temperature range of $26-32{ }^{\circ} \mathrm{C}$ and relative humidity range $50 \%-60 \%$ ). Five independent reflection measurements were carried out for all surfaces of each mortar set $\left(N_{1}=5 \times 4 \times 3=60\right.$ measurements for the lateral surfaces and $N_{2}=5 \times 3=15$ measurements for the bottom (and top) surfaces of the mortar sample M1 (and M2)) to obtain reliable results. All remaining details about sample preparation and curing conditions are available in [13].

\section{Measurement and discussion}

Fig. 1 shows the experimental setup operating between $3.95 \mathrm{GHz}$ and $5.85 \mathrm{GHz}$ (WR187) for reflection measurement of prepared samples. It involves a vector network analyzer (VNA) (Keysight Instruments - N9918 A) and a one-meter phase-stable cable. Scattering (S-) parameter measurements were implemented with 201 points. The setup was calibrated by the thru-reflect-line calibration technique prior measurements [17] by a line standard with length $20.51 \mathrm{~mm}$. It is noted that we selected the waveguide setup with the lowest cutoff frequency $\left(f_{c}=3.153 \mathrm{GHz}\right)$ in our laboratory, since microwave signals are very sensitive to variation of water content at microwave frequencies around $1 \mathrm{GHz}$ [18].

Frequency dependence of reflection response of samples $\Gamma$ is expressed by

$\Gamma=\frac{(1-g)+j b}{(1+g)-j b}$,

where $g$ shows the (normalized) conductance and $b$ indicates the (normalized) susceptance.

Figs. 2 and 3 illustrate the frequency dependencies of $|\Gamma|$ and $g$ values obtained from various sample surfaces with different $w / b$ ratios for 720th day of curing (hardened samples) after averaging measured $\Gamma(f)$ or $g(f)$ from each three set. Here, $|\star|$ denotes the magnitude of ' $\star$ '. Two important points are noted from the dependencies in Figs. 2 and 3. First, while frequency dependencies of $|\Gamma|$ or $g$ measurements from sample lateral surfaces with distinct $w / b$ are not distinguishable,

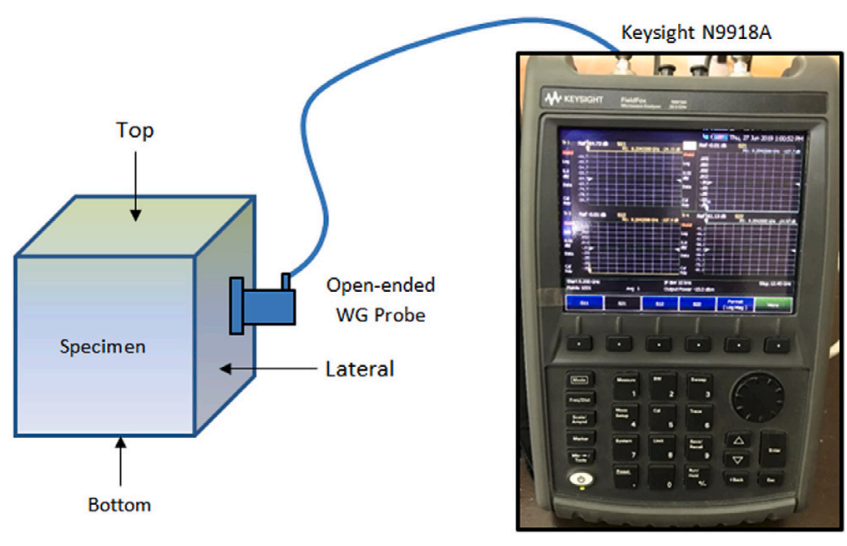

Fig. 1. Depiction of the experimental configuration.

there are considerable differences between frequency dependencies of $|\Gamma|$ (and $g$ ) measurements from bottom (or top) sample surfaces with distinct $w / b$. Second, $g$ measurements, as compared with $|\Gamma|$ measurements, have a wider span. It means that these differences can be easily monitored by $g$ changes. For example, while the difference between $|\Gamma|$ values from sample bottom surfaces with distinct $w / b$ at $3.85 \mathrm{GHz}$ is 0.011 , the difference between $g$ values is 0.118 .

On the other hand, we also examined temporal $g$ and $\Gamma$ dependencies of samples with different $w / b$ ratios at $4.56 \mathrm{GHz}$ over a long period of time (over 1-9 and 720-815 days) as shown in Figs. 4 and 5 after averaging measured $g(t)$ and $\Gamma(t)$ values. It is seen from Figs. 4(a), 4(d), $5(\mathrm{a})$, and $5(\mathrm{~d})$ that while $|\Gamma|$ value changes between 0.49 and 0.60 (0.47 and 0.69), $g$ value varies between 2.95 and 3.95 (2.70 and 5.20) for the mortar sample with lower (higher) $w / b$ ratio. Besides, similar to the conclusion we have drawn for frequency dependencies of $|\Gamma|$ and $g$ values in Figs. 2 and 3, because the range for $g$ was greater than that of $|\Gamma|$ for an analysis of reflection properties of mortar samples, measured $g$ results were fitted to the following power expression:

$g(t)=\xi_{1} t^{\xi_{2}}+\xi_{3}$,

where $t$ is the time variable in days and $a, b$, and $c$ are the constant coefficients. Evaluated $\xi_{1}, \xi_{2}$, and $\xi_{3}$ parameters for each ECM sample after fitting measurement results to the power expression in (2) are presented in Table 2. From the temporal dependencies of $g$ values for each sample in Figs. 4 and 5 and the data in Table 2, the following points are noted. As can be inferred from very high $R^{2}$ values (the higher the $R^{2}$ value the better the fitting $-R^{2}=1$ shows the best fitting), fitting parameters show good agreement between the fitted and measured $g$ values for different surfaces and $w / b$ ratios of ECM samples. Besides, while $g(t)$ quantities obtained from sample bottom surfaces with either $w / b$ ratio vary slowly with time, $g(t)$ quantities obtained from sample lateral or top surfaces with either $w / b$ ratio change very fast. Such an apparent difference arises from evaporation and hydration processes [19]. In addition, $g(t)$ quantities obtained from all sample surfaces with $w / b=0.2$ near 720 curing day are greater than the corresponding $g(t)$ quantities with $w / b=0.3$. As an example, whereas, at 720 curing day, the $g$ quantity obtained from the lateral sample surface with smaller $w / b$ is approximately 3.027, the $g$ quantity obtained from the lateral sample surface with higher $w / b$ is nearly 2.973. However, whereas the crossover between $g$ quantities measured from top (or lateral) sample surfaces with distinct $w / b$ occurs before 10th curing day [13], as shown in this study, the crossover between $g$ quantities obtained from sample bottom surfaces with distinct $w / b$ takes place around 550 days of curing (according to the fitted data). This is mainly due to fly ash [13] and displacement of heavier (e.g., sand) and lighter (e.g., bound-water) ingredients during hardening (setting) time, resulting in bleeding $[19,20]$. It is also noted 

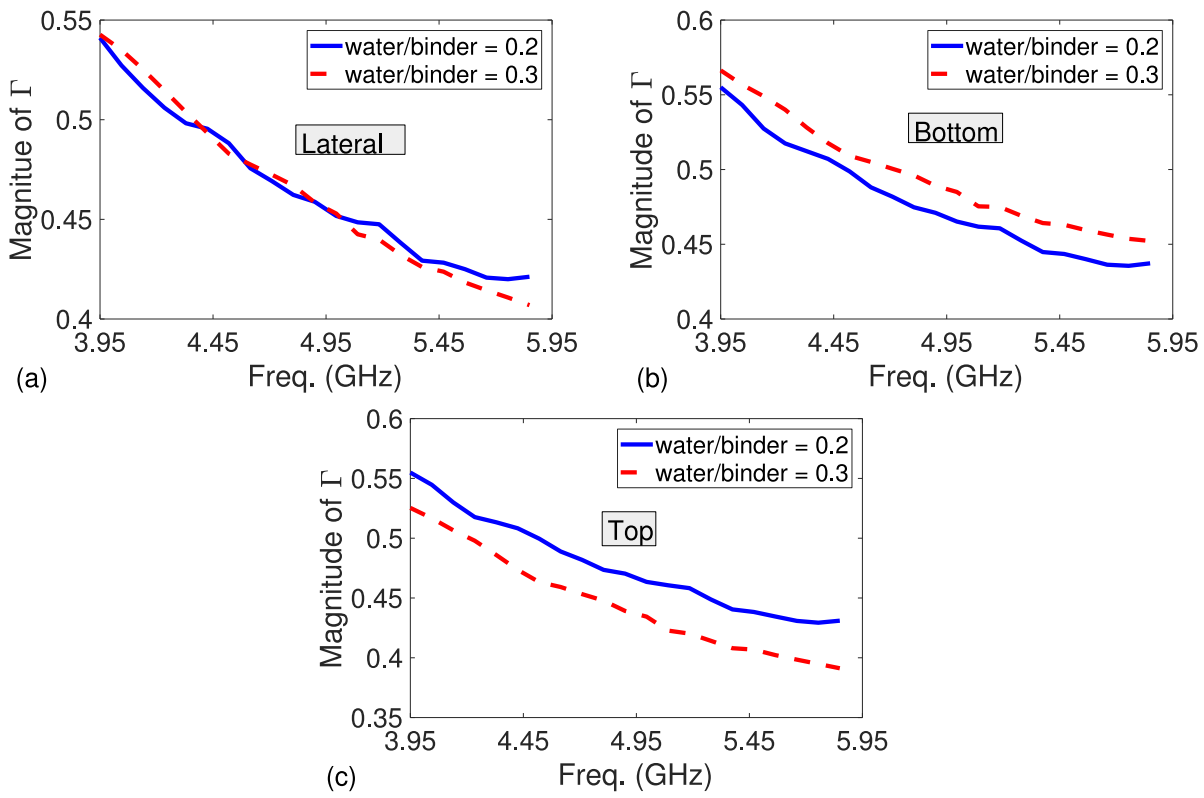

Fig. 2. Magnitude of reflection coefficient $\Gamma$ over frequency obtained from (a) lateral, (b) bottom, and (c) top surfaces of samples for 720 th day of curing.
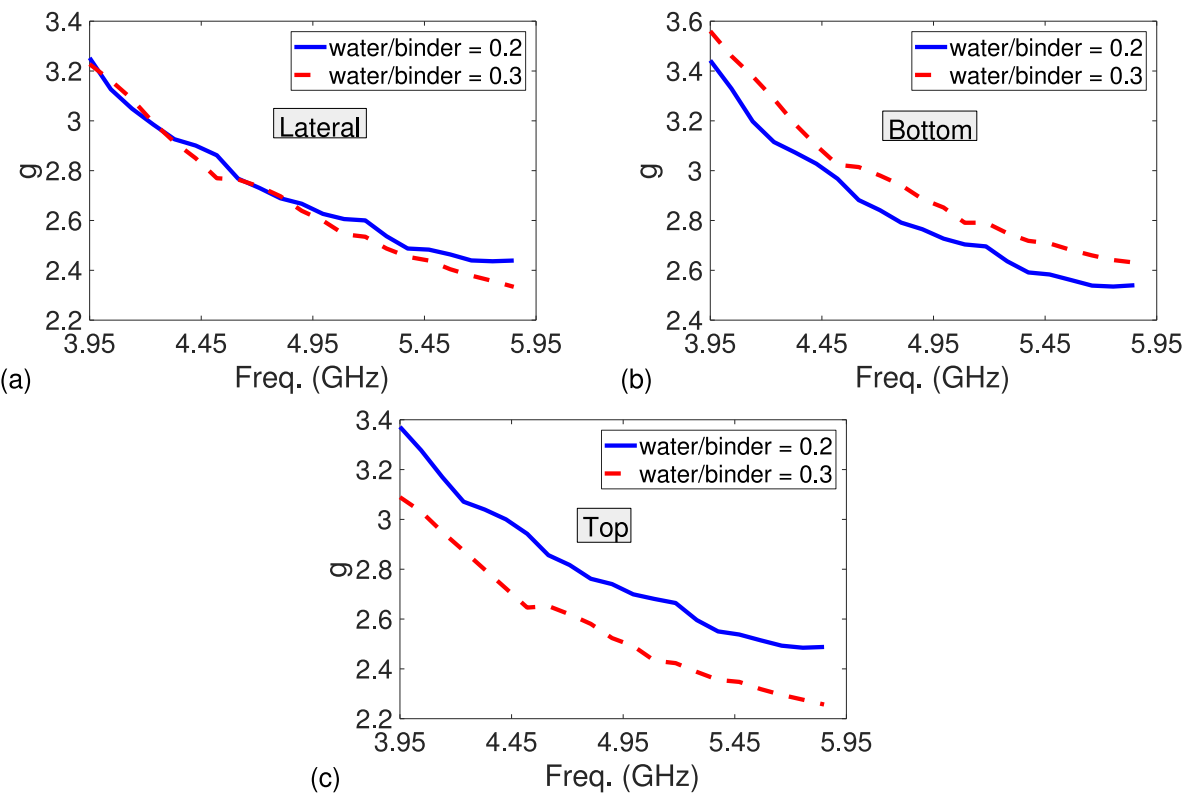

Fig. 3. Dependence of normalized conductance $g$ over frequency obtained from (a) lateral, (b) bottom, and (c) top surfaces of samples for 720 th day of curing.

that such a crossover was not observed for the magnitude of transmission properties measured from bottom surfaces of cement-based mortar samples with different water-to-cement ratios (0.4 and 0.7) over long curing periods [7]. Finally, it should be stated that different reflection properties depending on height of a large wall constructed by ECM samples, in addition to transmission properties [7], should be considered in propagation-related wireless local area networks [15].

Finally, it should be stated that reflection measurements were not carried out between 10 and 720 days, because we did not have access to the mortar samples due to pandemic issues. Nonetheless, higher $R^{2}$ values corresponding to each surface of both mortar samples could be considered as an indication that measurements between 10 and 720 days would follow the fitted parameters given in Table 2. Besides, reflection measurements $|\Gamma|$ and $g$ could suffer from non-sample-related signals such as signals arising from unexpected movements of the instrument. For such cases, soft computing methods such as the fuzzy
Table 2

Fitting metric along with fitting parameters for each sample.

\begin{tabular}{llllll}
\hline$w / b$ & Surface & $\xi_{1}$ & $\xi_{2}$ & $\xi_{3}$ & $R^{2}$ \\
\hline \multirow{3}{*}{0.2} & Bottom & 4.643 & -0.0379 & -0.614 & 0.9989 \\
& Lateral & 1.093 & -0.6736 & 2.858 & 0.9969 \\
& Top & 1.016 & -0.6416 & 2.927 & 0.9988 \\
\hline \multirow{3}{*}{0.3} & Bottom & -7.901 & 0.0356 & 12.92 & 0.9992 \\
& Lateral & 2.412 & -0.9325 & 2.773 & 0.9986 \\
& Top & 2.581 & -0.7753 & 2.630 & 0.9981 \\
\hline
\end{tabular}

neural approach could be a good promise [21]. As a future study, we want to perform a research on soft computing techniques for eliminating the effect of unexpected signals intermingled with the measured reflection signals. 

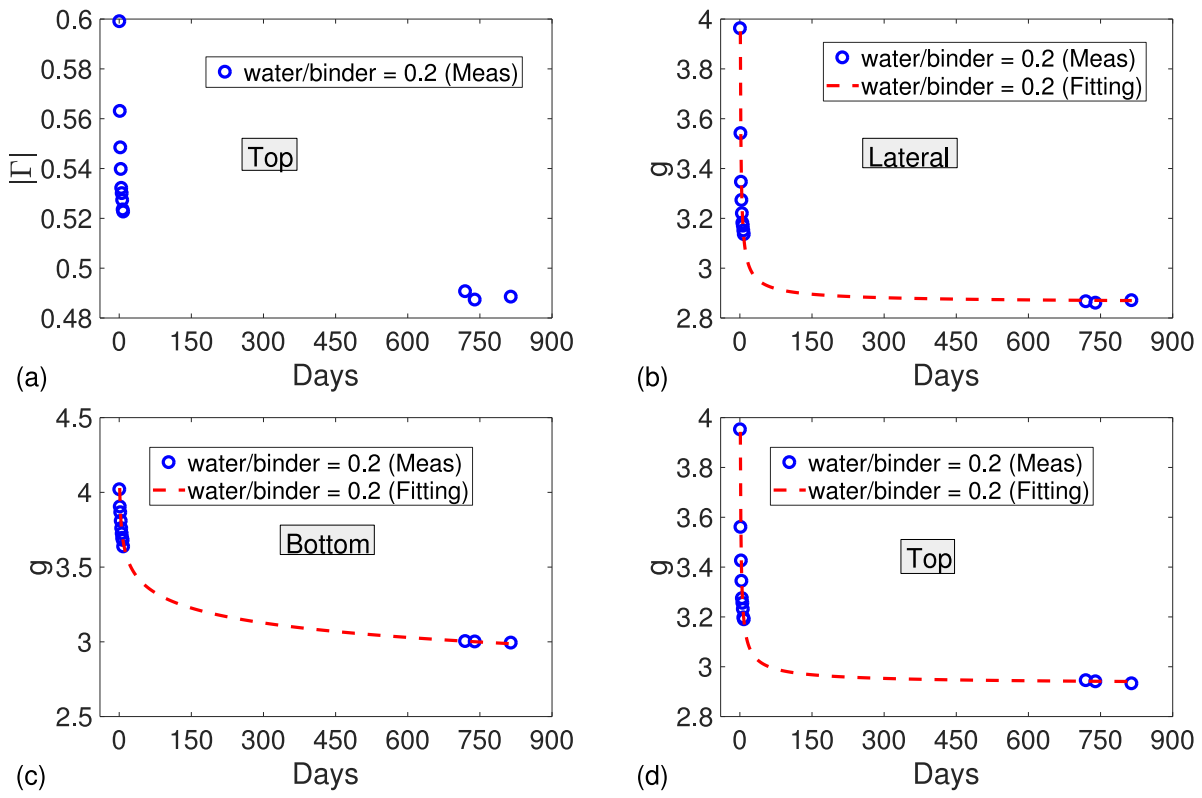

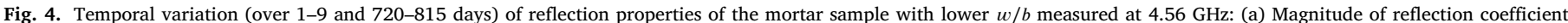
from the top surface and normalized conductance $g$ from (b) lateral, (c) bottom, and (d) top surfaces.
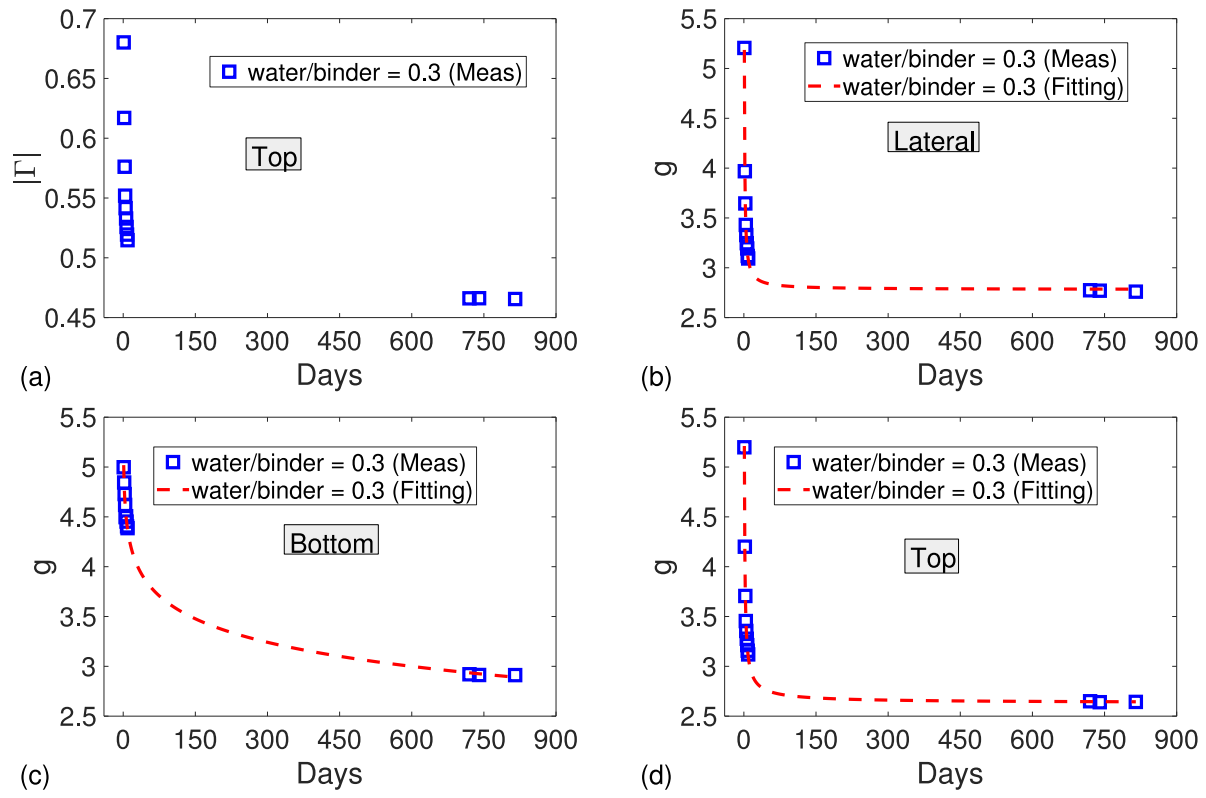

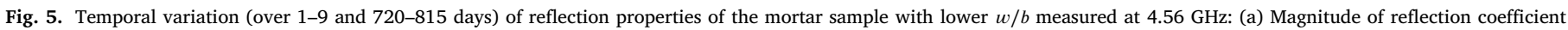
from the top surface and normalized conductance $g$ from (b) lateral, (c) bottom, and (d) top surfaces.

\section{Conclusion}

Microwave open-ended waveguide measurements were used to examine surface effects on reflection response of hardened ECM samples with distinct $w / b$. Temporal dependence of $g$ and $|\Gamma|$ values over long curing time (over 1-9 and 720-815 days) and frequency dependence of $|\Gamma|$ and $g$ values between $3.95-5.85 \mathrm{GHz}$ were examined for this goal. The following two points are noted from these dependencies. First, different than top and bottom surface behaviors, the crossover between $g$ quantities measured from bottom sample surfaces with lower and higher $w / b$ ratios delays for a long time (around 550 days). Second, reflection profiles of walls varying with height should be taken into account propagation-related studies for accurate prediction.

\section{CRediT authorship contribution statement}

Ugur Cem Hasar: Conceptualization, Methodology, Investigation, Writing - original draft. Mucahit Izginli: Visualization, Writing review \& editing. Hamdullah Ozturk: Visualization, Writing - review \& editing. Huseyin Korkmaz: Visualization, Writing - review \& editing. Abdulkadir Cevik: Investigation, Writing - review \& editing. Mohammad Refaat Irshidat: Writing - review \& editing.

\section{Declaration of competing interest}

The authors declare that they have no known competing financial interests or personal relationships that could have appeared to influence the work reported in this paper. 


\section{Acknowledgments}

U. C. Hasar and A. Cevik would like to thank the Scientific and Technological Research Council of Turkey (TUBITAK) under grant number 120M763 for providing the partial support to conduct our research. H. Ozturk acknowledges the TUBITAK BIDEB 2211/C program for supporting his studies.

\section{References}

[1] V.C. Li, On engineered cementitious composites (ECC): A review of the material and its applications, J. Adv. Concr Technol. 1 (5) (2003) 215-230.

[2] H. Ma, S. Qian, Z. Zhang, Effect of self-healing on water permeability and mechanical property of medium-early-strength engineered cementitious composites, Constr. Build. Mater. 68 (Oct.) (2014) 92-101.

[3] R. Zoughi, S.D. Gray, P.S. Nowak, Microwave nondestructive estimation of cement paste compressive strength, ACI Mater. J. 92 (1) (1995) 64-70.

[4] K.J. Bois, P.S.N.A. Benally, R. Zoughi, Microwave nondestructive determination of sand-to-cement ratio in mortar, J. Res. Nondestruct. Eval. 9 (4) (1997) $227-238$.

[5] K.J. Bois, A.D. Benally, P.S. Nowak, R. Zoughi, Cure-state monitoring and waterto-cement ratio determination of fresh portland cement-based materials using near-field microwave techniques, IEEE Trans. Instrum. Meas. 47 (3) (1998) 628-637.

[6] S.N. Kharkovsky, M.F. Akay, U.C. Hasar, C.D. Atis, Measurement and monitoring of microwave reflection and transmission properties of cement-based specimens, IEEE Trans. Instrum. Meas. 51 (6) (2002) 1210-1218.

[7] U. C.Hasar, Non-destructive testing of hardened cement specimens at microwave frequencies using a simple free-space method, NDT \& E Int. 42 (6) (2009) $550-557$.

[8] K.L. Chung, S. Kharkovsky, Monitoring of microwave properties of early-age concrete and mortar specimens, IEEE Trans. Instrum. Meas. 64 (5) (2015) 1196-1203.
[9] K.L. Chung, C. Song, Y. Li, C. Zhang, Correlation between microwave properties and compressive strength of engineered cementitious mortar, Microw. Opt. Technol. Lett. 58 (11) (2016) 2696-2699.

[10] K.L. Chung, C. Zhang, Y. Li, L. Sun, M. Ghannam, Microwave non-destructive inspection and prediction of modulus of rupture and modulus of elasticity of engineered cementitious composites (ECCs) using dual-frequency correlation, Sensors 17 (12) (2017) Art. no. 2831.

[11] K.L. Chung, J. Luo, L. Yuan, C. Zhang, C. Qu, Strength correlation and prediction of engineered cementitious composite with microwave properties, Appl. Sci. 7 (1) (2017) Art. no. 35.

[12] K.L. Chung, et al., Strength prediction and correlation of cement composites: A cross-disciplinary approach, IEEE Access 7 (2019) 41746-41756.

[13] U.C. Hasar, F. Bozdag, M. Bute, H. Ozturk, A. Cevik, Sample placement effect during curing on microwave reflection properties of early age engineered cementitious mortar specimens, IEEE Trans. Instrum. Meas. 69 (8) (2020) 5763-5771.

[14] K.L. Chung, L. Wang, M. Ghannam, M. Guan, J. Luo, Prediction of concrete compressive strength based on early-age effective conductivity measurement, J. Build. Eng. 35 (2021) 101998.

[15] I. Cuinas, M.G. Sanchez, Building material characterization from complex transmittivity measurements at 5.8GHz, IEEE Trans. Antennas Propag. 48 (8) (2000) 1269-1271.

[16] C. Li, T. Kanda, Engineered cementitious composites for structural applications, ASCE J. Mater. Civil Eng. 10 (1998) 66-69.

[17] G.F. Engen, C.A. Hoer, Thru-reflect-line: An improved technique for calibrationg the dual 6-port automatic network analyzer, IEEE Trans. Microw. Theory Techn. 27 (12) (1979) 983-987.

[18] A. Hashemi, M. Horst, K.E. Kurtis, K.M. Donnell, R. Zoughi, Comparison of alkalisilica reaction gel behavior in mortar at microwave frequencies, IEEE Trans. Instrum. Meas. 64 (7) (2015) 1907-1915.

[19] S. Popovics, Concrete Materials: Properties, Specifications, and Testing, Noyes Publications, New Jersey, 1992.

[20] P.K. Mehta, Concrete: Structure, Properties, and Materials, Prentice-Hall, New Jersey, 1986.

[21] E.C. Morabito, M. Versaci, A fuzzy neural approach to localizing holes in conducting plates, IEEE Trans. Magn. 37 (5) (2001) 3534-3537. 